\title{
Implementation of a Software Feedback Control for the CMS Monitoring Lasers
}

\author{
Liyuan Zhang, Member, IEEE, Kejun Zhu, David Bailleux, Adolf Bornheim, and \\ Ren-yuan Zhu, Senior Member, IEEE
}

\begin{abstract}
Light monitoring will play a crucial role in maintaining the energy resolution of the CMS lead tungstate crystal electromagnetic calorimeter (ECAL) in situ at LHC. Since 2001, a laser based monitoring system has been used in the CMS ECAL beam tests at CERN. While the stability of the laser pulse energy and width, measured in 24 hours, is at a level of $3 \%$, a long term degradation of both the laser pulse energy and the width were observed, as well as a drift of the laser pulse center timing at 2 ns/day. This degradation and drift, caused by the natural aging of the DC Kr lamp, would affect respectively the monitoring precision and the synchronization between the laser pulse and the ECAL ADC clock. This paper presents a design and implementation of a software feedback control which stabilizes the laser pulse energy, width and timing. With the software feedback implemented, a stability of the laser pulse energy and width at $3 \%$ level and a pulse timing jitter at 2 ns were observed in laser runs lasted for more than 3 months. The $0.5 \%$ energy resolution of the $\mathrm{CMS} \mathrm{PbWO}_{4}$ crystal ECAL is maintained after applying the laser monitoring corrections in a beam irradiation test.
\end{abstract}

Index Terms-Calorimeter, feedback control, laser monitoring, lead tungstate crystal, radiation damage.

\section{INTRODUCTION}

$\mathbf{T}$ HE main physics motivation for building the Compact Muon Solenoid (CMS) experiment at the Large Hadron Collider (LHC) is to investigate the mechanism responsible for the electroweak symmetry breaking. In the low mass range between 115 and $150 \mathrm{GeV}$ the Higgs discovery potential in the $\gamma \gamma$ decay channel is directly related to the reconstructed mass width, or the energy resolution of the electromagnetic calorimeter (ECAL). The CMS lead tungstate $\left(\mathrm{PbWO}_{4}\right)$ crystal ECAL has a designed energy resolution

$$
\frac{\sigma E}{E}=\frac{\mathbf{2 . 7} \%}{\sqrt{E}} \oplus \mathbf{0 . 5 5} \% \oplus \frac{0.16}{E}
$$

for the barrel and

$$
\frac{\sigma E}{E}=\frac{\mathbf{5 . 7} \%}{\sqrt{E}} \oplus \mathbf{0 . 5 5} \% \oplus \frac{0.77}{E}
$$

for the endcaps, where $\oplus$ stands for addition in quadrature and $E$ is in $\mathrm{GeV}$ [1]. Test beams at CERN have shown that this energy

Manuscript received December 7, 2006; revised November 16, 2007. This work was supported in part by the U.S. Department of Energy under Grant DE-FG03-92-ER-40701 and in part by the U.S. National Science Foundation Awards PHY-0612805 and PHY-0516857.

The authors are with the California Institute of Technology, Pasadena, CA 91125 USA (e-mail:zhu@hep.caltech.edu).

Color versions of one or more of the figures in this paper are available online at http://ieeexplore.ieee.org.

Digital Object Identifier 10.1109/TNS.2007.914024

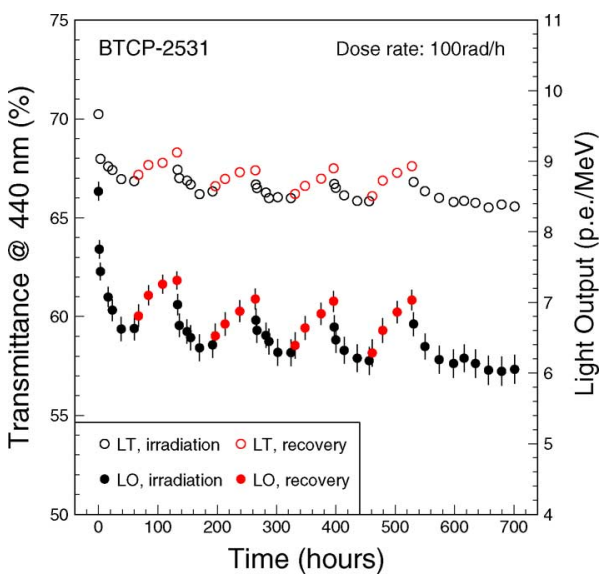

Fig. 1. Variations of the longitudinal transmission (left scale, open circles) and the light output (right scale, solid dots) are shown as a function of time for a full size CMS PbWO 4 crystal BTCP-2531 in a multi-cycle irradiations of $100 \mathrm{rad} / \mathrm{h}$ followed by recoveries.

resolution can be achieved by using mass-produced $\mathrm{PbWO}_{4}$ crystals with avalanche photodiode (APD) readout [2]. A crucial issue, however, is to maintain this energy resolution, especially the $0.55 \%$ constant term, in situ at LHC.

The 75,848 $\mathrm{PbWO}_{4}$ crystals in the CMS ECAL would suffer from radiation damage in situ at LHC [1]. Because of the high energy and the high luminosity expected at LHC the CMS detector will be operated in a severe radiation environment. The mass produced lead tungstate crystals are radiation hard to a high integrated dose, but suffer from a dose rate dependent radiation damage as shown in the radiation induced absorption [3]. Our previous studies concluded that the scintillation mechanism of $\mathrm{PbWO}_{4}$ crystals is not affected by the radiation, and the loss of the light output is due only to the absorption caused by the radiation induced color centers [4]. A precise calibration in situ thus is crucial for maintaining the energy resolution offered by a precision crystal calorimeter. During the LHC runs, inter-calibrations will be carried out by using physics events, such as electrons from $\mathrm{Z}$ or $\mathrm{W}$ decays and photon pairs from $\pi^{0}$ and $\eta$ decays. During the time needed to accumulate sufficient statistics, however, $\mathrm{PbWO}_{4}$ crystals would experience radiation damage (beam on) and recovery (beam off) shown in the variations of crystal's transparency. To achieve $0.5 \%$ inter-calibration precision these variations must be corrected.

Fig. 1 shows a history of the light output (solid dots) and the longitudinal transmission at $440 \mathrm{~nm}$ (open circles) measured for a full size CMS $\mathrm{PbWO}_{4}$ crystal sample in a series of damage and recovery cycles. The irradiations were carried out by using a ${ }^{60} \mathrm{Co} \gamma$-ray source at a dose rate of $100 \mathrm{rad} / \mathrm{h}$, 


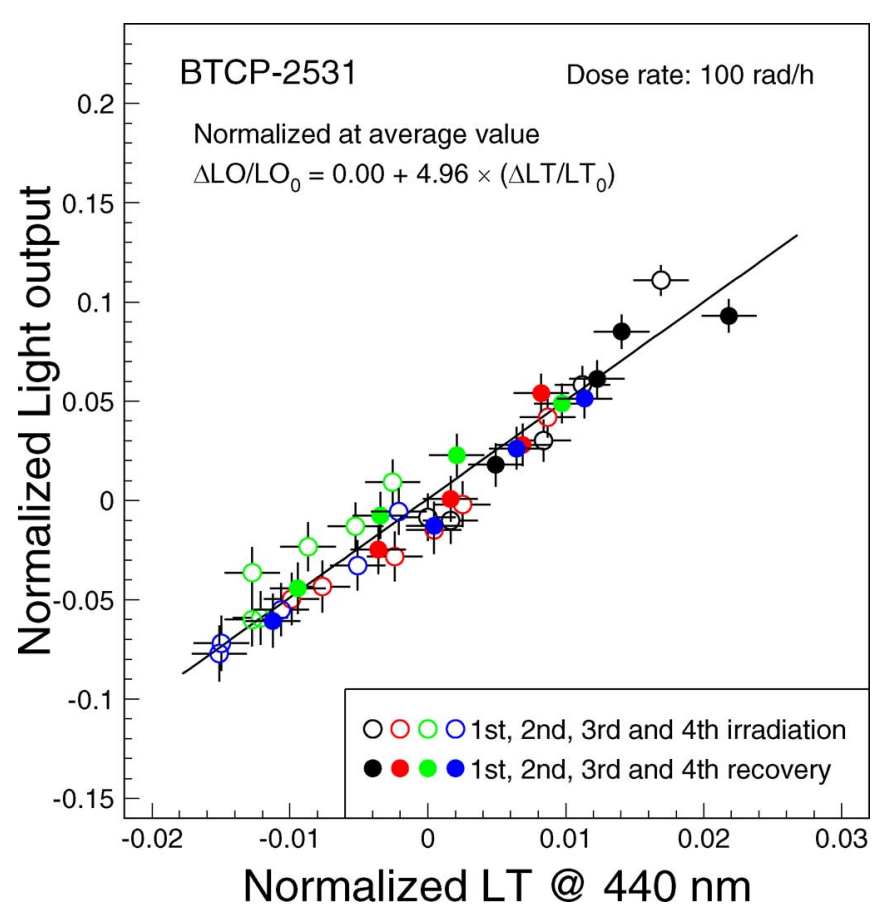

Fig. 2. The normalized variations of the light output are shown as a function of the normalized variations of the longitudinal transmission at $440 \mathrm{~nm}$ for sample BTCP-2531 under $100 \mathrm{rad} / \mathrm{h}$ irradiation.

which is expected by the CMS ECAL endcaps running at the designed luminosity [1]. The observed variations are caused by the color center formation (damage) and annihilation (recovery) processes [4]. Fig. 2 presents a linear fit to the data shown in Fig. 1. The excellent linearity indicates that the variations of crystal's light output may be calculated and therefore corrected by using the variations of crystal's longitudinal transmittance at $440 \mathrm{~nm}$.

For the CMS $\mathrm{PbWO}_{4}$ calorimeter, a light monitoring system was designed and constructed by the Caltech and Saclay groups [1]. Its design goal is to measure the variations of crystal's optical transparency and use that to make corrections for the variations of crystal's light output [5]. Fig. 3 is a schematic showing the laser based CMS light monitoring system. The monitoring laser pulses produced by one of three pairs of lasers are distributed via an optical fiber system organized into three stages. A $1 \times 88$ fiber optic switch sends laser pulses to one of 88 calorimeter elements ( 72 half super modules in the barrel and 16 groups of super crystals in two endcaps). A two stage distribution system mounted on each calorimeter element delivers monitoring laser pulses to each individual crystals. While the laser pulses are injected at the front face of the barrel crystals, they are injected at the back face of the endcap crystals where space is available. The laser pulse intensity measured by the ECAL readout device APDs is normalized to that measured by the reference PN diode. The APD/PN ratio is the monitoring signal, which is a measure of crystal's transparency. The stability of APD/PN ration is required to be $0.2 \%$ to achieve the $0.5 \%$ inter-calibration precision.

In this paper we present the design of the CMS monitoring laser light source. A software feedback control, which improves the stability of the laser performance significantly, is also described. The energy resolutions of the CMS $\mathrm{PbWO}_{4}$ crystals, obtained in an irradiation test, without and with the laser monitoring corrections are also shown to demonstrate the effectiveness of the monitoring corrections.

\section{MOnitoring LASER System}

The light source and high level distribution subsystem (LSDS) was designed and constructed at Caltech. The top plot of Fig. 4 is a schematic showing the design of LSDS. As shown in this figure, it consists of three pairs of lasers, a $3 \times 1$ optical switch, an $1 \times 88$ optical switch, a fast monitor, a logarithmic attenuator, a linear attenuator and a laser PC running a laser DAQ control program. Each pair of lasers consists of a Nd:YLF pump laser, a tunable Ti:Sapphire laser and a digital scope based slow monitor [6]. The bottom plot of Fig. 4 is a schematic showing the details of one laser pair.

All three pump lasers are model 527DQ-S Q-switched $\mathrm{Nd}$ :YLF lasers, which are a commercial product of Quantronix [7]. It provides frequency doubled laser pulses at $527 \mathrm{~nm}$ with a pulse intensity up to $20 \mathrm{~mJ}$ at a repetition rate up to $15 \mathrm{kHz}$. All three Ti:Sapphire lasers are custom made Proteus UV(SHG) lasers from Quantronix, which provide a laser pulse intensity up to $1 \mathrm{~mJ}$, corresponding to about $1.3 \mathrm{TeV}$ energy deposition in $\mathrm{PbWO}_{4}$ crystals, at a repetition rate up to $100 \mathrm{~Hz}$. Two wavelengths are available from each pair of lasers by selecting appropriate built-in interference filters in the Ti:Sapphire laser. $440 \mathrm{~nm}$ was chosen as the monitoring wavelength since it provides the best linearity between the variations of crystal's light output and its transmittance [8].

Two pairs of the lasers provide $440 \mathrm{~nm}$ (blue) and $495 \mathrm{~nm}$ (green), so that the $440 \mathrm{~nm}$ light is always available even when one laser pair is in maintenance. The third pair provides $709 \mathrm{~nm}$ (red) and $796 \mathrm{~nm}$ (IR) for monitoring the gain variations of the readout electronics chain from the APD to the ADC, where the variations of crystal's transparencies are much more reduced. Only one laser pair is used at a time to provide monitoring laser pulses, which is selected by using the DiCon $3 \times 1$ optical switch. By using the DiCon $1 \times 88$ optical switch the monitoring laser pulses are sent to one of the 88 detector elements with each consisting of about 850 (barrel) or 916 (endcaps) crystals. Two attenuators are installed in the attenuation box between two switches. A logarithmic filter wheel allows an attenuation between 0 to $50 \mathrm{db}$ in $10 \mathrm{db}$ steps. A linear neutral density filter provides an additional attenuation between $1 \%$ to $99 \%$ in $1 \%$ step. The operation time needed to scan all 88 monitoring blocks is about 20 minutes. During LHC runs, laser pulses are sent to each detector elements in the LHC beam gaps of $3.17 \mu \mathrm{s}$, which are designed to rest the LHC kicker magnet in every $89.924 \mu \mathrm{s}$. Only about $1 \%$ of the available beam gaps are used for the ECAL monitoring data taking during the LHC operation with corresponding monitoring data rate of about $100 \mathrm{~Hz}$.

The entire laser operation is under the control of a laser PC which runs the laser DAQ program. It selects the laser pair, the ECAL detector element, the laser pulse wavelength and the intensity attenuation. All these parameters may also be defined by an up-level laser supervisor when the LSDS is running in a slave mode. In addition, the laser PC also controls internal laser 


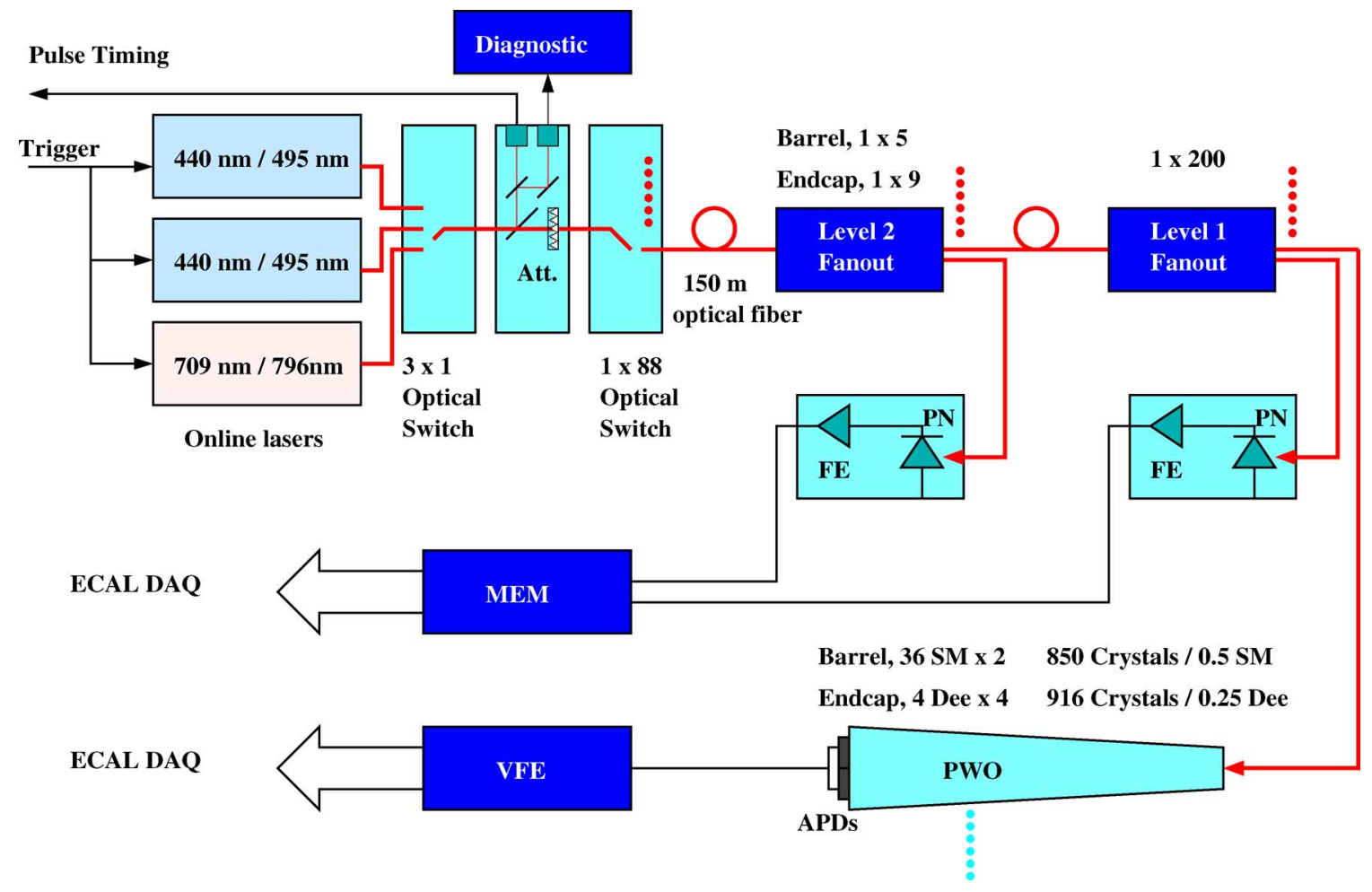

Fig. 3. A schematic showing the CMS laser monitoring system.

parameters, such as the YLF pumping current, the digital delays between various laser triggers and the Agilent digital scope based slow monitor, which collect laser pulse data at a sampling rate of $2 \mathrm{GS} / \mathrm{s}$. The slow monitor is capable to analyze the laser pulses from both the Nd:YLF laser and the Ti:Sapphire laser at a rate up to $1 \mathrm{~Hz}$. An Acqiris DP210 digitizer card is inserted between two optical switches. It functions as a fast monitor, and is capable to analyze every laser pulse delivered to the detector at $100 \mathrm{~Hz}$ with a sampling rate of $2 \mathrm{GS} / \mathrm{s}$. Laser pulse energy, width (FWHM) and pulse center timing are analyzed on-line during laser operation by both the slow and fast monitors, and the resultant histories of these parameters and the histograms of all measured laser pulses are stored. While the slow monitor data taking are controlled by the laser DAQ PC, the fast monitor data taking are controlled by another dedicated PC as shown in Fig. 4.

The first blue/green laser system was installed and commissioned at CERN in August, 2001 [8]. The IR/red laser system and the second blue/green laser system were installed and commissioned at CERN in August, 2003 [9]. The LSDS system has been in operation since 2001 for the ECAL beam tests and cosmic tests [2]. For typical laser runs lasted for about $24 \mathrm{~h}$, the stability of the laser pulse energy and width was found to be at a level of 3\% much better than the $10 \%$ specification [6]. For runs beyond 24 hours a degradation of the stability and a drift of the laser pulse timing at a level of 2 ns/day were observed. This is due to the aging of the DC Kr lamp used to pump the Nd:YLF laser [10]. While the degraded stability may affect the monitoring precision, the laser timing drift may affect the synchronization between the laser pulses and the LHC bunch train and the ECAL ADC clock in situ at LHC. To address this issue a software feedback control was implemented in 2006, which compensate the aging of the DC Kr lamp by trimming the Nd:YLF laser pumping current.

\section{SofTWARE FEEDBACK}

Figs. 5 and 6 show the measured correlations between the Nd:YLF laser pumping current and the laser pulse energy, FWHM and pulse center timing for the Nd:YLF laser and the Ti:Sapphire laser respectively. Also shown in these figures are the numerical values of the slope defined at the Nd:YLF laser pumping current of $25 \mathrm{~A}$. It is clear that when the Nd:YLF laser pumping current increases both the laser pulse width and the pulse center timing decrease and the laser pulse energy increases. This is understood since the optical gain of the Nd:YLF crystal increases when the pumping current increases, so the built-up time of the laser pulses in the laser cavity decreases and thus a shorter pulse.

In principle, any parameter or a combination of these parameters can be used as the input for the feedback to trim the Nd:YLF laser pumping current and thus compensate the aging of the DC $\mathrm{Kr}$ lamp. Looking into the slope values of these parameters in Figs. 5 and 6 and their sensitivities as compared to the corresponding r.m.s. values of the parameter, however, the Ti:Sapphire laser pulse center timing is preferred.

A software feedback control was implemented in the laser DAQ program. Fig. 7 is a schematic showing the software feedback control. Fig. 8 shows the control panel and software feedback setting window running under the laser DAQ program. When the software feedback is enabled, the program first calculates an average of a selected parameter, e.g., the pulse center 


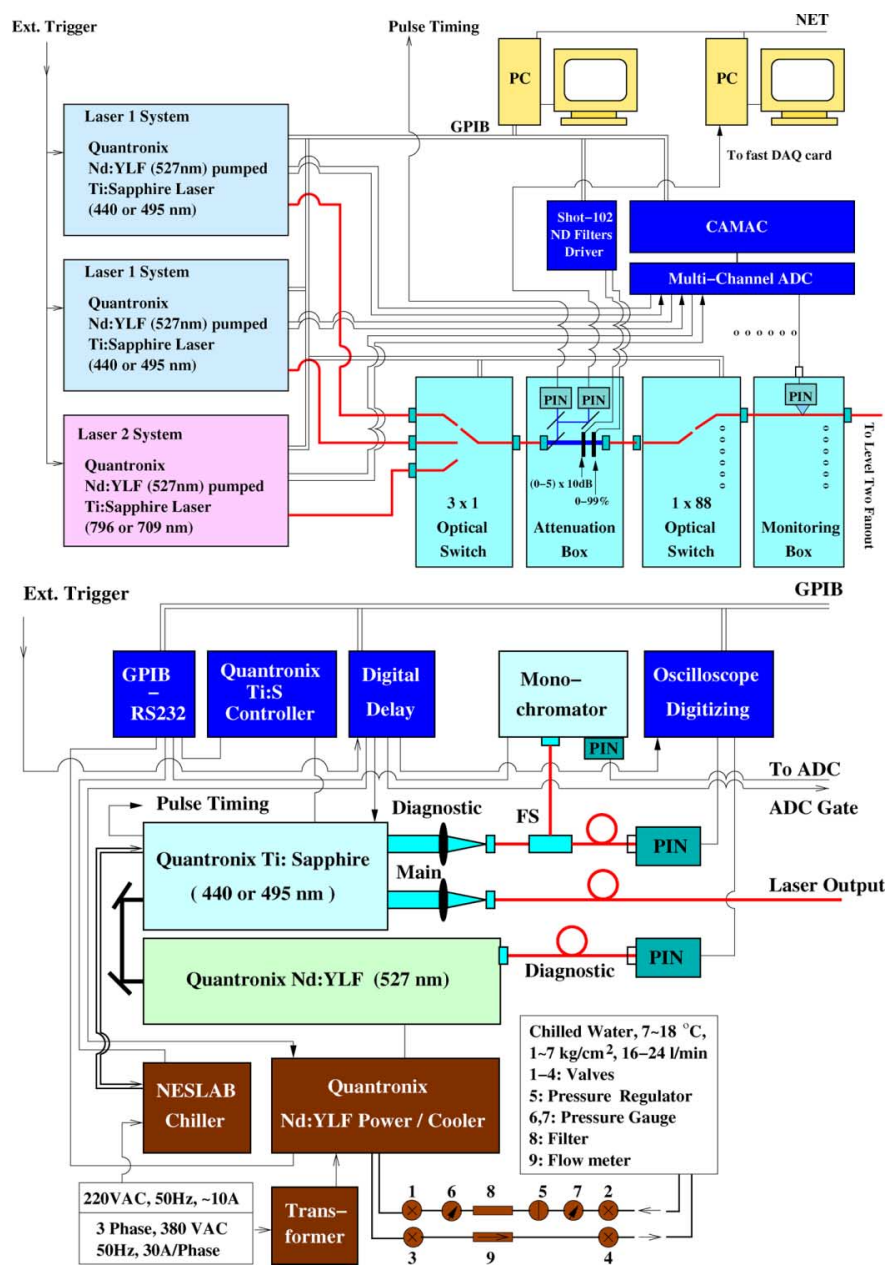

Fig. 4. A schematic (top) showing the design of the laser light source and the high level distribution system (LSDS) with the details of one laser system shown at the bottom.

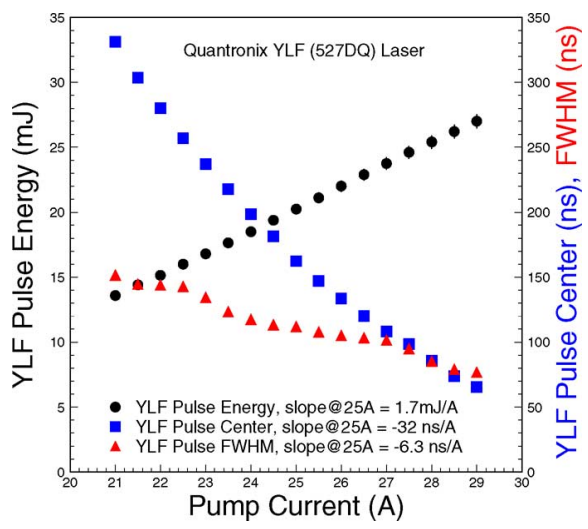

Fig. 5. Correlations between the Nd:YLF laser pumping current and the Nd:YLF laser pulse energy, width (FWHM) and pulse center timing.

timing, for a defined number of laser pulses, e.g., 600. This average is then compared to a predefined value of the pulse center timing from a data file, and their difference is used to calculate an incremental values of the Nd:YLF laser pumping current. If its absolute value is larger than $0.1 \mathrm{~A}$, then the Nd:YLF laser's pumping current is trimmed correspondingly, otherwise not. This step of $0.1 \mathrm{~A}$ is limited by the Quantronix Nd:YLF

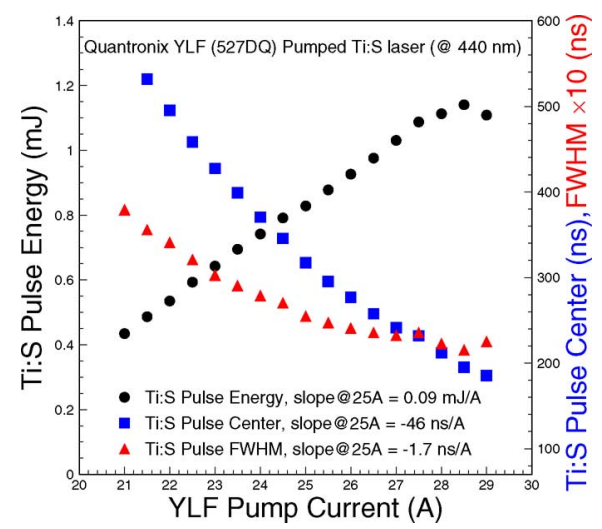

Fig. 6. Correlations between the Nd:YLF laser pumping current and the Ti:Sapphire laser pulse energy, width (FWHM) and the pulse center timing.

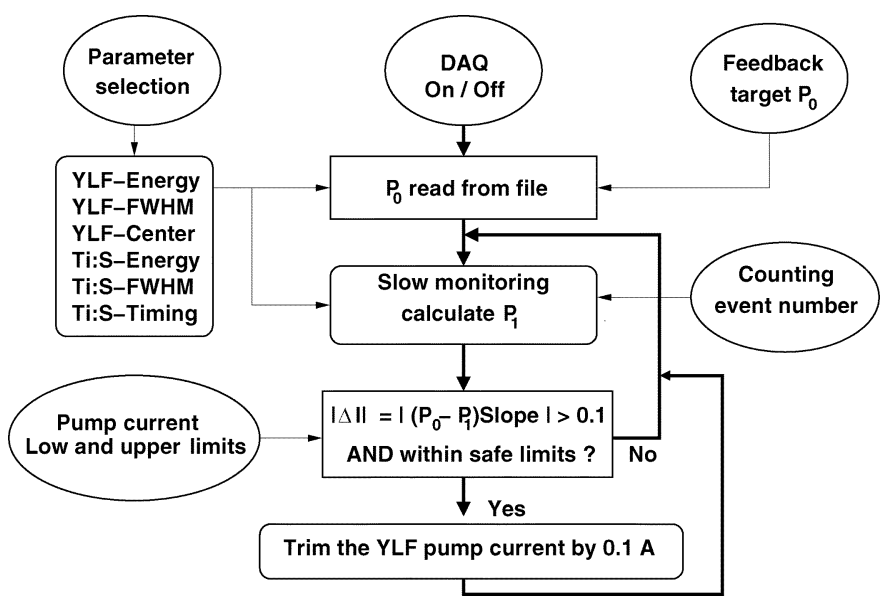

Fig. 7. A schematic describing the software feedback control.

laser control hardware. Upper and low limits of the Nd:YLF laser pumping current are also set for laser safety. The Nd:YLF laser pumping current will stay unchanged when it reaches the upper limit, indicating that the DC Kr lamp needs to be replaced.

\section{LASER PERFORMANCE}

The software feedback control was first implemented for individual laser runs during the ECAL beam tests in 2006. Figs. 9 and 10 summarize typical laser performance at $440 \mathrm{~nm}$ for two laser runs without and with the software feedback control lasting for 130 and 180 hours respectively. The left side of these plots are the histories of laser pulse energy (arbitrary unit), FWHM (ns), pulse center timing (ns) and corresponding YLF pumping current. The pulse center timing is the delay from the trigger to the laser pulse timing defined as the laser pulse intensity weighted average timing. Its typical value is about $3.5 \mu \mathrm{s}$.

The right side of these plots are corresponding histograms of these parameters with entries of all measured laser pulses. The r.m.s. spread represents the real distribution. As shown in Fig. 10, a significant narrow distributions of these parameters were observed by using the Ti:Sapphire pulse center timing as the feedback parameter. The r.m.s. spreads of the laser pulse energy, width and timing were reduced from $2.3 \%$ to $1.4 \%, 3.2 \%$ 


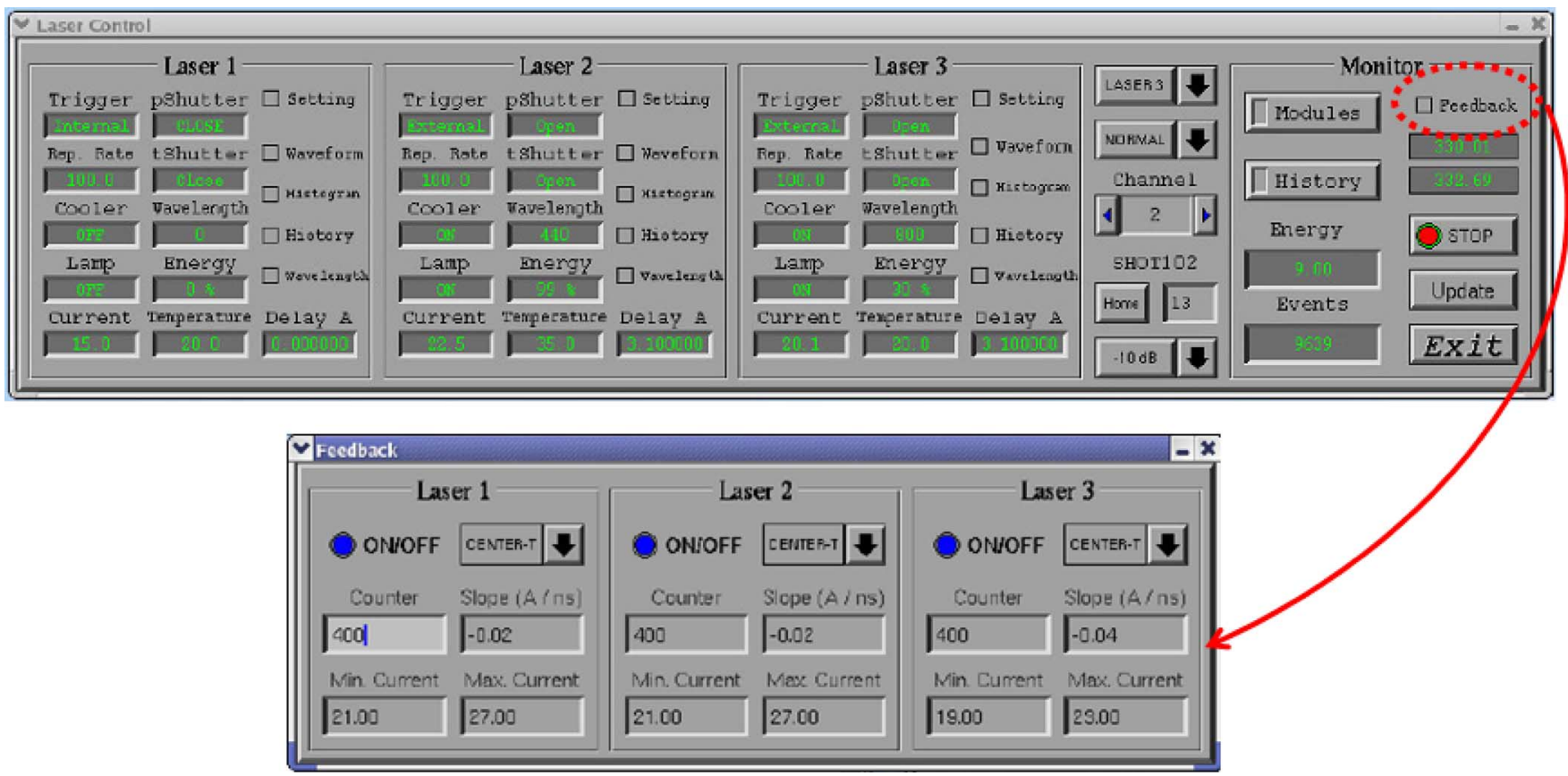

Fig. 8. The graphic interface of the laser DAQ control panel (top) and the feedback setting window (bottom).
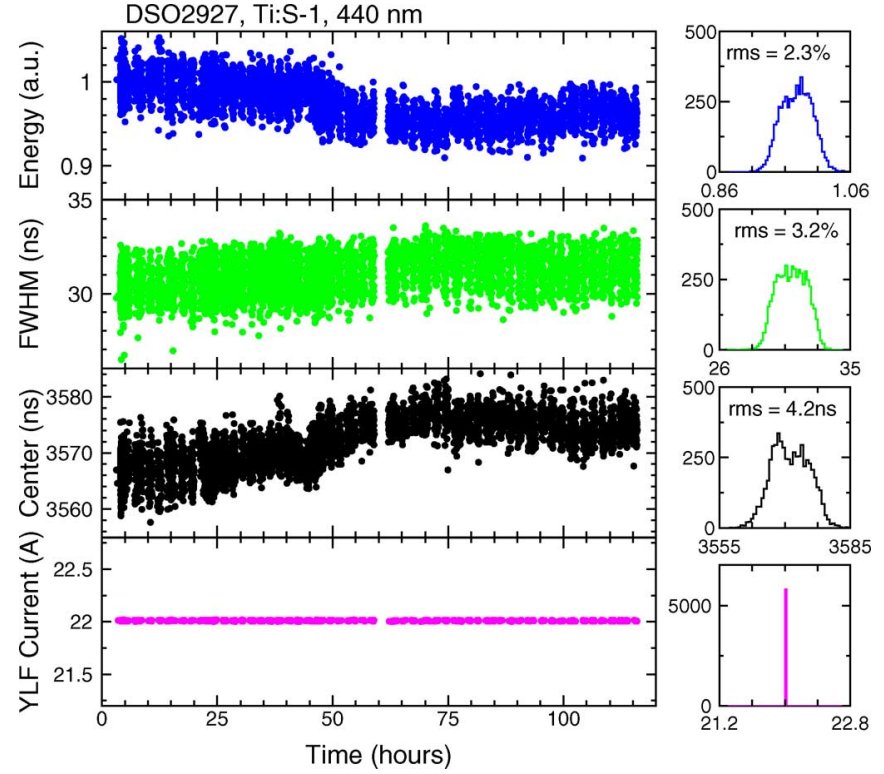

Fig. 9. The history of the laser pulse energy, width (FWHM), pulse center timing and the corresponding YLF laser pumping current is shown as function of time for a Ti:Sapphire laser run at $440 \mathrm{~nm}$ without the feedback for more than 130 hours. The corresponding histogram distributions are shown at the right.

to $2.6 \%$ and $4.2 \mathrm{~ns}$ to $1.8 \mathrm{~ns}$ respectively while the YLF pumping current was increased from $22.4 \mathrm{~A}$ to $22.7 \mathrm{~A}$. .

Please note, these data were taken during the ECAL beam test, where the laser pulse generation is not continuous although the DC $\mathrm{Kr}$ lamp is turned on. This is due to the fact that laser pulses were generated only when the ECAL test beam module is ready to accept them. This explains the gap at $60 \mathrm{~h}$ observed in Fig. 9 when the ECAL supermodules are changed. Similar gaps are observed in Fig. 10.

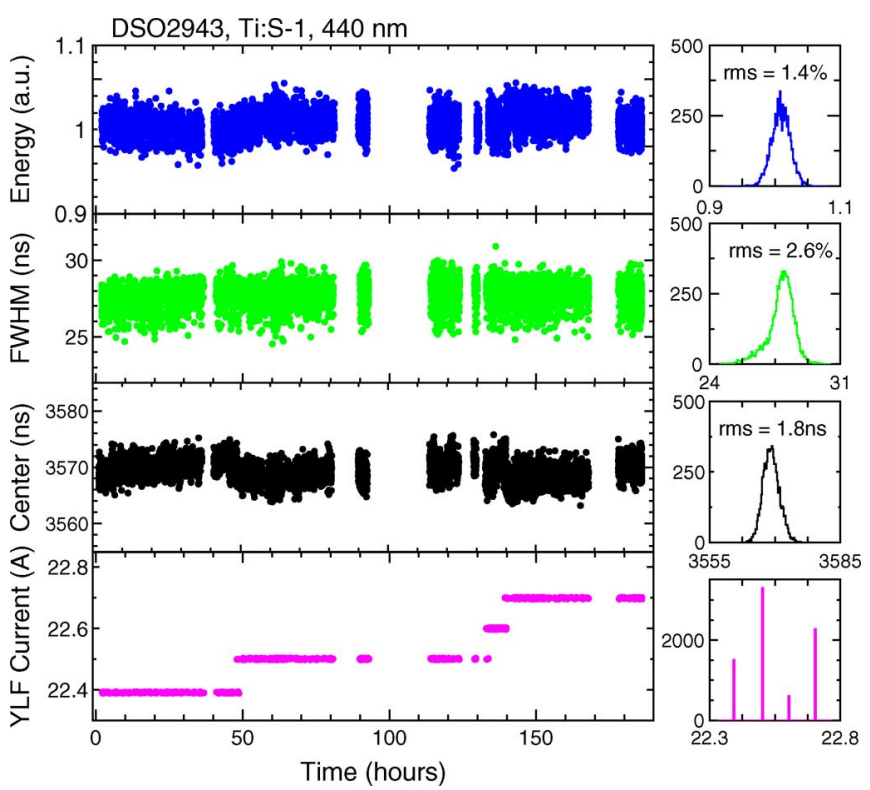

Fig. 10. The same as Fig. 9 for a Ti:Sapphire laser run at $440 \mathrm{~nm}$ for more than 180 hours by using the Ti:Sapphire laser pulse timing as the software feedback parameter.

Fig. 9 also shows a shift of laser performance between 45 to $60 \mathrm{~h}$. This shift is related to a temperature variation in the laser barracks at the CERN test beam site. Although the Ti:Sapphire laser's SHG crystal is thermally stabilized by a Neslab chiller (see the bottom plot of 4), variations of the room temperature would slightly change the Ti:S SHG crystal's matching angle, thus compromise the SHG crystal's conversion efficiency. Such shift may also occur when water is filled into the Neslab cooler, which changes the stabilized temperature by $0.5^{\circ} \mathrm{C}$.

Environmental temperature dependence of the laser performance was measured. The slope was found to be $-4.3 \% /{ }^{\circ} \mathrm{C}$, 


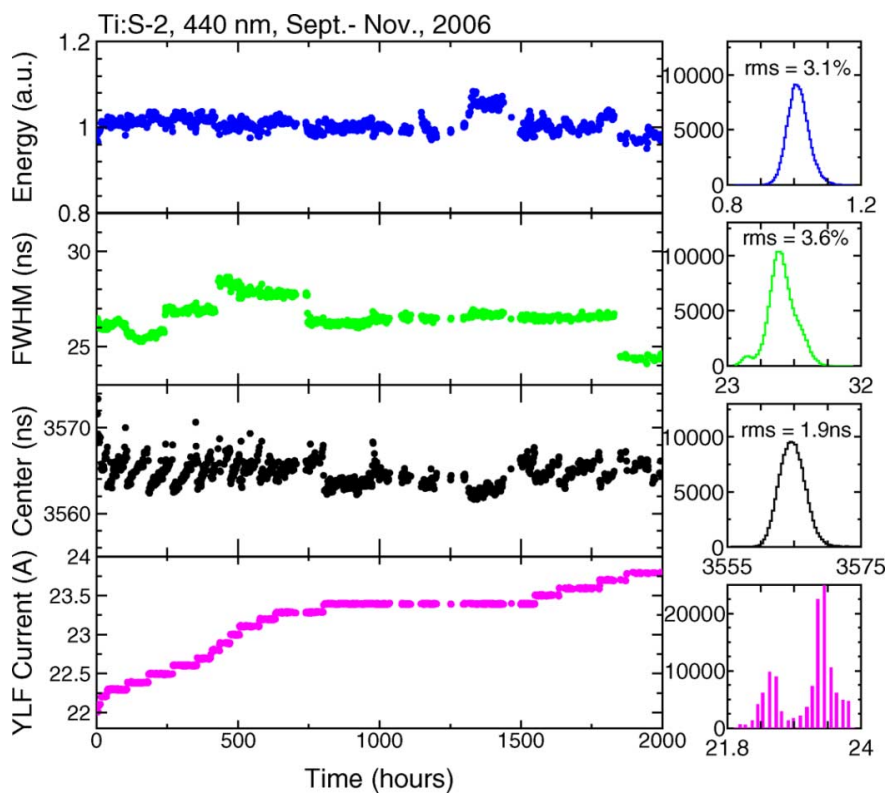

Fig. 11. The same as Fig. 9 for combined Ti:Sapphire laser runs at $440 \mathrm{~nm}$ for more than 3 months.

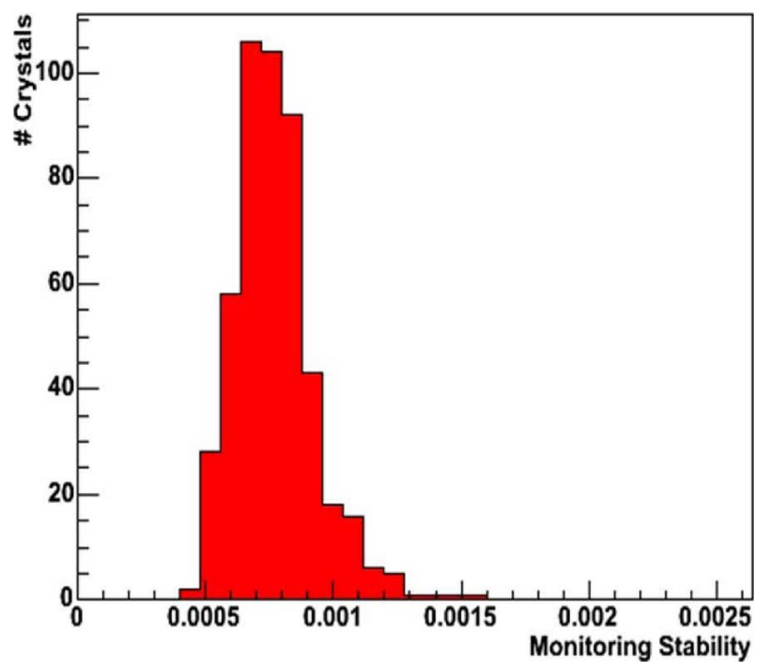

Fig. 12. The monitoring system stability shows a $0.1 \%$ is achieved in the ECAL beam test.

$1.3 \mathrm{~ns} /{ }^{\circ} \mathrm{C}$ and $7.9 \mathrm{~ns} /{ }^{\circ} \mathrm{C}$ respectively for the laser pulse energy, width (FWHM) and pulse timing. While the temperature stability is poor in the laser barracks at the CERN test beam site, it will be improved in situ at LHC, where the laser barracks located in the CMS underground carven are central air-conditioned with a temperature stability expected to be better than $0.5^{\circ} \mathrm{C}$. The data shown in Figs. 9-11 are not corrected for the temperature variations.

By using a pre-defined laser pulse timing, which is 3,565 ns from the trigger Fig. 11 shows a history and histogram for combined laser runs at $440 \mathrm{~nm}$ with a total run time more than three months. The history of the laser pulse energy, width, center timing and the YLF pumping current is shown at the left side of Fig. 11, where each data point represents an one hour average. During this period the Nd:YLF pumping current was increased automatically from 22 A to 23.8 A to compensate the
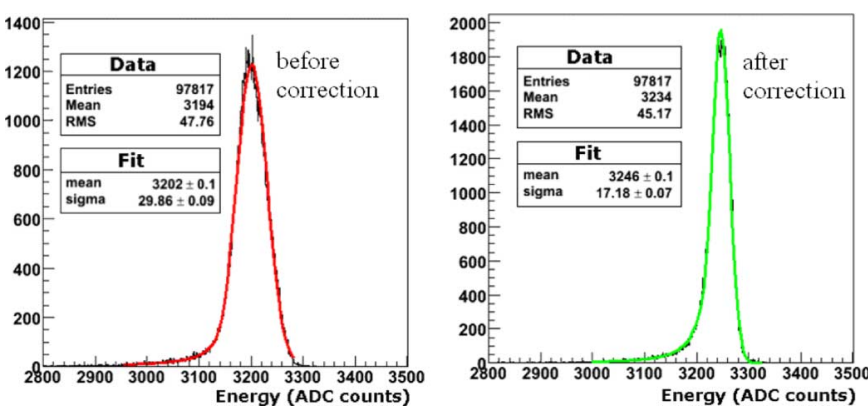

Fig. 13. The energy resolutions of $120 \mathrm{GeV}$ electrons, reconstructed by summing $3 \times 3$ crystals in an irradiation study, are shown before (left) and after (right) the monitoring corrections.

lamp aging. The histogram plots at the right shows distributions for all laser pulses analyzed during this period. The effectiveness of the software feedback control is clearly shown in the distribution of the laser pulse timing. The overall stability of the laser pulse energy and FWHM observed is about $3.1 \%$ and $3.6 \%$ respectively, much better than the $10 \%$ specification. The corresponding pulse jitter during this period is $1.9 \mathrm{~ns}$, much better than the $3 \mathrm{~ns}$ specification. We also notice four shifts in the laser pulse width (FWHM) history at 220, 420, 750 and 1,700 hours, which are related to the temperature variations and the refilling of the Neslab cooler as discussed above.

\section{ECAL RESOLUTIONS}

With the software feedback implemented, the stability of the APD/PN monitoring signal was observed at a level of $0.1 \%$ [2] as shown in Fig. 12, which is much better than the $0.2 \%$ specification [1]. The performance of the monitoring system was demonstrated in an irradiation study carried out during the 2006 beam test. Fig. 13 shows that the energy resolution for $120 \mathrm{GeV}$ electrons, reconstructed by summing $3 \times 3$ crystals, are $0.93 \%$ and $0.53 \%$ respectively before and after applying the monitoring corrections. This indicates that the $0.5 \%$ energy resolution of $\mathrm{PbWO}_{4}$ crystals may be maintained by applying the laser monitoring corrections.

\section{SUMmary}

Excellent linearity was observed between the variations of the light output and the variations of the longitudinal transmittance at $440 \mathrm{~nm}$ for the $\mathrm{PbWO}_{4}$ crystals. Because of the natural aging of the DC Kr lamp used by the Nd:YLF pump laser the performance of the Ti:Sapphire lasers show a long term degradation. A software feedback control was implemented to compensate this aging effect by trimming the Nd:YLF laser pumping current. The result shows a significant improvement in the laser stability. The stability of the laser pulse energy and FWHM width have been maintained at a level of 3\%, and the laser pulse center timing jitter has been reduced to less than 2 ns in laser runs lasting for more than three months. The overall laser monitoring precision is maintained at a level of less than $0.1 \%$. The energy resolution of the CMS $\mathrm{PbWO}_{4}$ ECAL in situ at LHC is expected to be maintained by applying the laser monitoring corrections. 


\section{ACKNOWLEDGMENT}

The authors would like to thank the CMS ECAL group, especially members of Caltech and Saclay groups, for fruitful collaborations and many useful discussions.

\section{REFERENCES}

[1] "The Electromagnetic Calorimeter Technical Design Report," CMS collaboration, 1997, CERN/LHCC 97-33.

[2] P. Adzic et al., "Results of the first performance tests of the CMS electromagnetic calorimeter," Eur. Phys. J. C, vol. 44, no. 2, pp. 1-10, 2006.

[3] R. H. Mao, L. Y. Zhang, and R. Y. Zhu, "Quality of mass produced lead tungstate crystals," IEEE Trans. Nucl. Sci., vol. 51, no. 4, pp. 1777-1783, Aug. 2004.
[4] R.-Y. Zhu, "Radiation Damage in Scintillating Crystals," Nucl. Instr. and Meth., vol. A413, pp. 297-311, 1998.

[5] X. D. Qu, L. Y. Zhang, and R. Y. Zhu, "Radiation induced color centers and light monitoring for lead tungstate crystals," IEEE Trans. Nucl. Sci., vol. 47, no. 6, pp. 1741-1747, Dec. 2000.

[6] L. Y. Zhang, D. Bailleux, A. Bornheim, K. J. Zhu, and R. Y. Zhu, "Performance of the monitoring light source for the CMS lead tungstate crystal calorimeter," IEEE Trans. Nucl. Sci., vol. 52, no. 4, pp. 1123-1130, Aug. 2005.

[7] Quantronix, East Setauket, NY [Online]. Available: http://www. quantronixlasers.com.

[8] L. Y. Zhang, K. J. Zhu, R. Y. Zhu, and D. Liu, "Monitoring light source for CMS lead tungstate crystal calorimeter at LHC," IEEE Trans. Nucl. Sci., vol. 48, no. 3, pp. 372-378, Jun. 2001.

[9] D. Bailleux, A. Bornheim, L. Y. Zhang, K. J. Zhu, R.-Y. Zhu, and D. Liu, "ECAL Monitoring Light Source at H4," 2003, CMS IN 2003/045.

[10] L. Y. Zhang, R.-Y. Zhu, and D. Liu, "Monitoring Lasers for PWO ECAL," 1999, CMS IN 1999/014. 